\title{
High-pressure diamondlike liquid carbon
}

\author{
Luca M. Ghiringhelli, ${ }^{1}$ Jan H. Los, ${ }^{2}$ Evert Jan Meijer, ${ }^{1}$ A. Fasolino, ${ }^{1,2}$ and Daan Frenkel ${ }^{1,3}$ \\ ${ }^{1}$ van 't Hoff Institute for Molecular Sciences, Universiteit van Amsterdam, Nieuwe Achtergracht 166, \\ 1018 WV Amsterdam, The Netherlands \\ ${ }^{2}$ Theoretical Physics, NSRIM, University of Nijmegen, Toernooiveld, 6525 ED Nijmegen, The Netherlands \\ ${ }^{3}$ FOM Institute for Atomic and Molecular Physics, Kruislaan 407, 1098 SJ, Amsterdam, The Netherlands
}

(Received 31 October 2003; published 12 March 2004)

\begin{abstract}
We report density-functional based molecular-dynamics simulations, which show that, with increasing pressure, liquid carbon undergoes a gradual transformation from a liquid with local threefold coordination to a "diamondlike" liquid. We demonstrate that this unusual structural change is well reproduced by an empirical bond-order potential with isotropic long-range interactions, supplemented by torsional terms. In contrast, state-of-the-art short-range bond-order potentials do not reproduce this diamond structure. This suggests that a correct description of long-range interactions is crucial for a unified description of the solid and liquid phases of carbon.
\end{abstract}

DOI: 10.1103/PhysRevB.69.100101

PACS number(s): 61.20.Ja, 34.20.Cf, 71.15.Pd

When a simple fluid, such as argon, is cooled below the critical temperature, it can form a liquid phase. However, more complex fluids, such as molten phosphorus, can form two distinct liquid phases that are separated by a first-order liquid-liquid phase transition (LLPT). ${ }^{1}$ There is increasing evidence that a variety of network-forming liquids can undergo an LLPT (see, e.g., Franzese et l. $^{2}$ ). In particular, it has long been suspected that elemental carbon may exhibit an LLPT. ${ }^{3,4}$ As direct experiments are difficult in the relevant temperature regime (4000-6000 K), much of the evidence for such a transition comes from simulations. Classical simulations based on the Brenner bond-order potential with torsional terms ${ }^{5,6}$ predicted a coexistence line between a twofold and a fourfold coordinated liquids starting from the melting line of graphite end ending in a critical point (at $\sim 8800 \mathrm{~K})^{7}$ as shown in Fig. 1. Recent first-principle investigations ${ }^{8}$ have ruled out the occurrence of such a transition in the range of specific volumes $6.59-15.6 \AA^{3} /$ atom at $6000 \mathrm{~K}$. More importantly, these $a b$ initio results found a majority of three-fold coordination over the studied range. Wu et al. ${ }^{8}$ pointed out that the presence of $\pi$ bonds in the liquid phase makes it difficult to represent the torsion potential adequately. They concluded that empirical potentials are unable to capture these subtle electronic effects.

In this Rapid Communication, we reexamine these issues by extending the range of studied densities while comparing density-functional theory based molecular dynamics (DFMD) simulations to Monte Carlo (MC) results based on two state-of-the-art empirical potentials. The first is the recently proposed long-range carbon bond-order potential (LCBOP) (Ref. 9) extended with torsional interactions; the second is the reactive empirical bond-order (REBO) (Ref. 10) potential which improves the earlier version due to Brenner. ${ }^{5}$ LCBOP introduces long-range terms to account for interplanar binding of graphite which is not described by the REBO potential.

On the basis of DF-MD we find that, while no true LLPT occurs even for higher densities than in Wu et al., ${ }^{8}$ a well defined switching of the dominant coordination from three to four is found at $\sim 5.8 \AA^{3} /$ atom, just beyond the range stud- ied in Wu et al. ${ }^{8}$ This "diamondlike" fourfold coordinated liquid is highly structured, with a strongly anisotropic angular distribution of the first neighbors. Interestingly, the empirical potential LCBOP gives an impressive agreement with the DF-MD results for the structural properties of this highdensity liquid whereas the short-ranged REBO potential ${ }^{10}$ yields a graphitelike liquid in this range. It should be noted that both the low-density threefold coordinated and highdensity diamondlike liquid are very unusual. In fact most covalent semiconductors become highly coordinated in the liquid phase. ${ }^{14}$ Highly coordinated phases have been predicted by Grumbach and Martin ${ }^{15}$ also for carbon albeit at pressures of TPa.

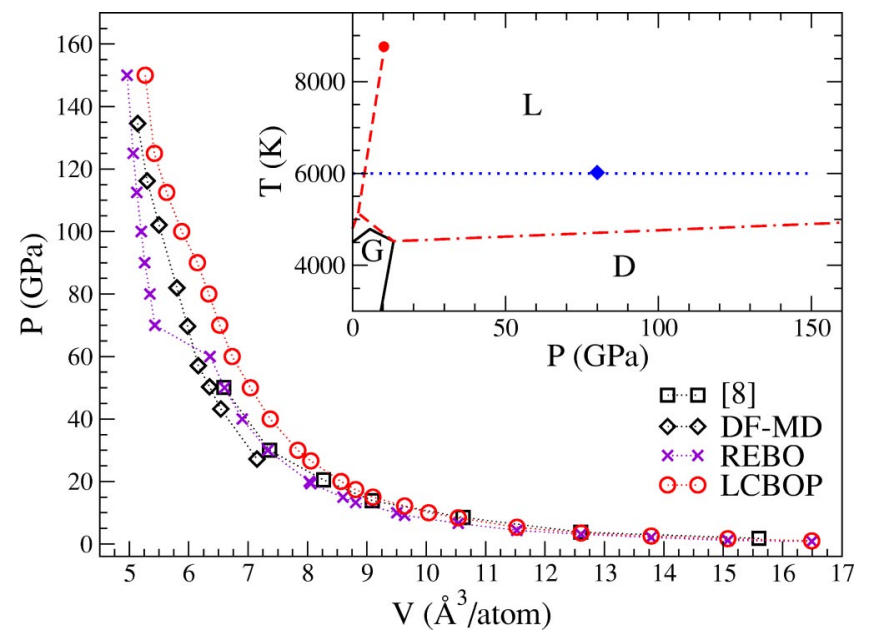

FIG. 1. (Color online) Equation of state of liquid carbon at $T$ $=6000 \mathrm{~K}$. The inset shows the $P-T$ phase diagram, with diamond (D), graphite $(\mathrm{G})$, and liquid (L) domains: the dotted curve is the sampled isotherm, the solid curves are experimental data-the diamond-graphite coexistence curve from Bundy Ref. 11, the graphite melting curve from Togaya (Ref. 12). The dot-dashed line is the estimated diamond melting line from Glosli and Ree (Ref. 13), obtained with Brenner potential, the dashed curves are the liquid-liquid coexistence curve ending in a critical point and its graphite melting curve from Glosli and Ree (Ref. 7). The $\downarrow$ in the inset indicates the switching in coordination shown in Fig. 2. 
We performed constant volume DF-MD simulations using the Car-Parrinello method ${ }^{16}$ as implemented in CPMD package ${ }^{17}$ The system consisted of 128 atoms in a cubic box with periodic boundary conditions at nine densities and a temperature $T=6000 \mathrm{~K}$, imposed by means of a Nosé-Hoover ${ }^{18}$ thermostat. We used the Becke ${ }^{19}$ exchange and Perdew ${ }^{20}$ correlation gradient corrected functional (BP) with a plane-wave basis set cut off at $35 \mathrm{Ry}$ and sampled the Brillouin zone only in the gamma point. BP gives a correct description of bulk diamond. Each state point was studied for 5 ps, starting from a sample equilibrated via LCBOP; only minor structural changes occurred in the first tenths of ps. Since liquid carbon is metallic, we imposed a thermostat for the electronic degrees of freedom in order to ensure a proper implementation of the Car-Parrinello scheme. ${ }^{21}$ We performed the MC simulations of 128 particles in a cubic box with periodic boundary conditions with the LCBOP and the REBO potential. We sampled at $6000 \mathrm{~K}$ the constant volume ensemble for specific volumes larger than $8 \AA^{3}$ /atom and the constant pressure ensemble for smaller specific volumes where the increase of pressure is steeper, with an overlap between the two regions to check for consistency. MC simulations at selected volumes with 512 and 4096 particles using the LCBOP showed negligible differences with the 128 particle results for the local structure and equation of state.

In order to reproduce the $a b$ initio results, we needed to refine the LCBOP. ${ }^{9}$ The detailed procedure will be described elsewhere. ${ }^{22}$ Here we give a brief summary of the changes. Following the strategy of the REBO potential ${ }^{10}$ we have introduced a correction to the angular function in order to stabilize small clusters in shapes other than chainlike. It is in fact known (see, e.g., Raghavachari and Binkley ${ }^{23}$ ) that, while odd numbered clusters prefer to arrange in a chain, even numbered ones can also have metastable ring or cage structures. We focused attention on a planar $C_{4}$ cluster with $C_{2 v}$ symmetry (a rhombus with two $\sim 60^{\circ}$ angles ${ }^{23}$ ) and a cubic $C_{8} .{ }^{24}$ It turned out that the angular dependence of the bond order had to be weakened for coordination lower than three. More important is the addition of torsional interactions, in such a way as to describe the conjugation dependence of the torsional energy barrier in agreement with the $a b$ initio calculations for double and conjugated bonds of $\mathrm{Wu}$ et $a l .{ }^{8}$ This dependence is not captured by the REBO potential. There, the barrier as a function of the dihedral angle $\omega_{i j k l}$ is always described by $\sin ^{2}\left(\omega_{i j k l}\right)$ and differs only by a scale factor between these two bonding situations. In the original LCBOP, a variable $N_{i j}^{c o n j}$ was already defined, assuming values 1 or 0 , respectively, in these two extreme cases, to account for the presence or absence of a $\pi$ bond. We fit the results of Wu et al. ${ }^{8}$ for $N_{i j}^{c o n j}=0,1$ by two polynomials in $\cos ^{2}\left(\omega_{i j k l}\right)$ and interpolate between them by means of a function decaying rather quickly away from $N_{i j}^{c o n j}=1$. The torsional energy is activated only for a bond between threefold coordinated atoms, as for the REBO potential. The smaller torsional energy of a bond between fourfold coordinated atoms is already taken into account by the long-range interactions between the third neighbors.

In Fig. 1 we compare the pressure-volume isotherms of our DF-MD simulations with our MC results based on the

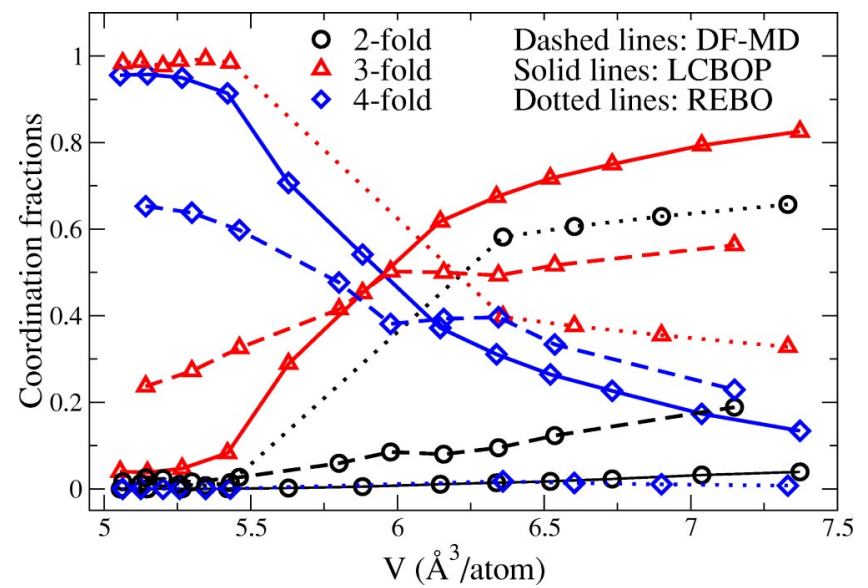

FIG. 2. (Color online) Coordination fractions vs specific volume of liquid carbon. Note that at $V \sim 6 \AA^{3} /$ atom DF-MD and LCBOP predict a switch from threefold to fourfold coordination, whereas REBO evolves from a twofold to a graphitelike liquid.

empirical REBO and LCBOP potentials, and with the DF-MD data of Wu et al., ${ }^{8}$ all at a temperature of $6000 \mathrm{~K}$. In view of the relatively low cutoff ( $35 \mathrm{Ry}$ ), we had to correct the pressures for the spurious contribution due to Pulay forces. ${ }^{25}$ In the density range where we can compare with the results of $\mathrm{Wu}$ et al., ${ }^{8}$ the pressures that we compute are some $15 \%$ lower than those reported by $\mathrm{Wu}$ et al. This relatively small difference is probably due to the different choice of density functionals. We have checked that our samples were indeed liquid. Over the whole isotherm we have observed diffusive behavior in both the MC-LCBOP and the DF-MD simulations, the latter indicating a self-diffusion coefficient at least of order $10^{-5} \mathrm{~cm}^{2} / \mathrm{s}$.

Up to $60 \mathrm{GPa}$ both empirical potentials seem to be in fair agreement with $a b$ initio data. However, the coordination fractions shown in Fig. 2 indicate that the REBO potential fails to reproduce the correct liquid structure. It predicts, in the range between 6 and $7.5 \AA^{3} /$ atom, mainly twofold coordination, whereas the present DF-MD results (and those of Wu et $a l .{ }^{8}$ ), as well as the LCBOP, show dominant threefold coordination and low twofold coordination.

At higher pressures, DF-MD predicts a marked switching of dominant coordination from three to four around $5.8 \AA^{3} /$ atom. Judging from the isotherm of Fig. 1, the transition seems to be continuous with no sign of a van der Waals loop. These results are consistent with the tightbinding MD simulations of Morris et al. ${ }^{26}$ In contrast, between 5.6 and $6.0 \AA^{3}$ /atom, where the switch of dominant coordination takes place, the MC results based on LCBOP display large fluctuations in density at the imposed pressure of $100 \mathrm{GPa}$, resulting in a slight bending of the isotherm of Fig.1. Again, qualitatively the coordination fractions obtained by LCBOP agree with the DF-MD results even though with a more pronounced switch to coordination four. Curiously, the REBO potential predicts a first-order phase transition in the same density range but to a completely different structure, a threefold graphitelike liquid with well ordered sliding sheets which eventually get stuck upon further in- 


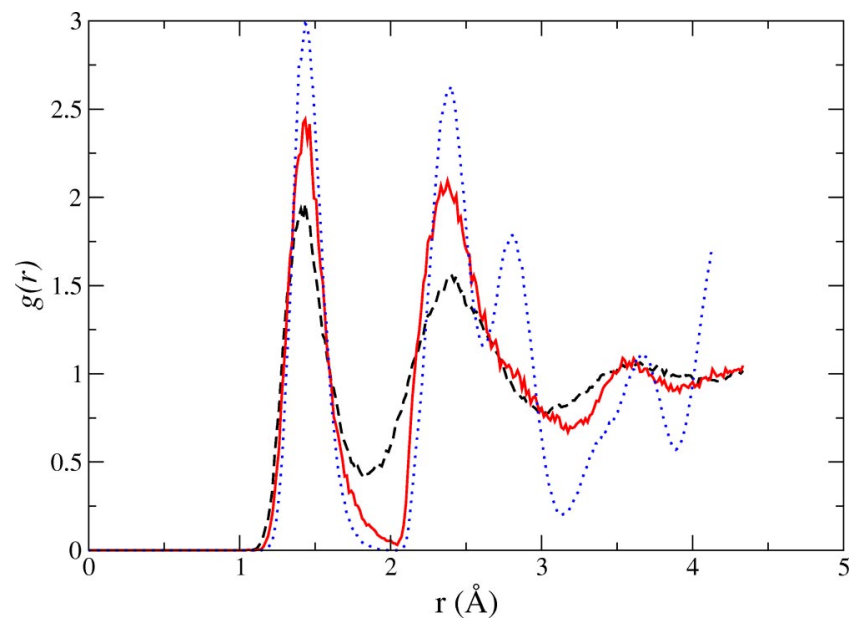

FIG. 3. (Color online) Pair-correlation functions at 5000 K. Diamond (dotted curve) is at $150 \mathrm{GPa}$; the LCBOP liquid (solid curve) is at $150 \mathrm{GPa}$ and $5.14 \AA^{3}$ /atom; DF-MD liquid (dashed curve) is at $5.14 \AA^{3}$ /atom.

creasing of the pressure. The REBO potential never gives rise to fourfold coordination.

If the threefold to fourfold coordination change extrapolates to lower temperatures, it might provide an explanation for the sudden change of the slope of the graphite melting line. ${ }^{12}$ The origin of the latter is still an open question, although it has been suggested that it is associated with a firstorder phase transition. ${ }^{4,12}$ Our results provide no evidence of a first-order transition but rather indicate a pronounced but continuous change of dominant coordination.

Both the DF-MD and LCBOP simulations of the highdensity fourfold coordinated liquid show a strong diamond like positional and orientational order, as shown in Figs. 3-5. In Fig. 3, we compare the pair correlations $g(r)$ of this highdensity liquid with that of a (meta)stable bulk diamond near the estimated coexistence point, at $5000 \mathrm{~K}$ and $150 \mathrm{GPa}$ (also the LCBOP and DF-MD liquid samples were equilibrated at that temperature). One can see that up to the second neighbor shell the liquid has a structure almost as pronounced as dia-

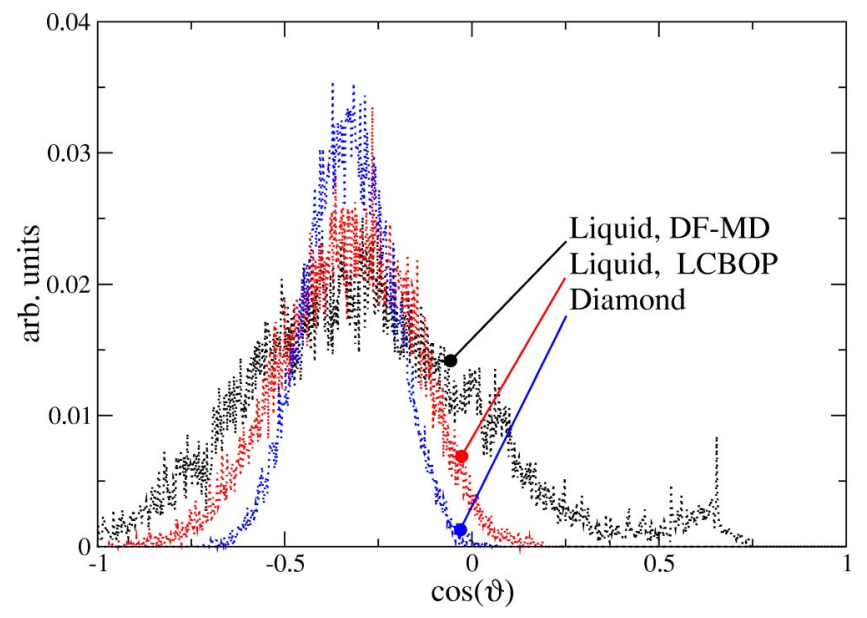

FIG. 4. (Color online) Angular correlation functions of first neighbors with state parameters as in Fig. 3 .

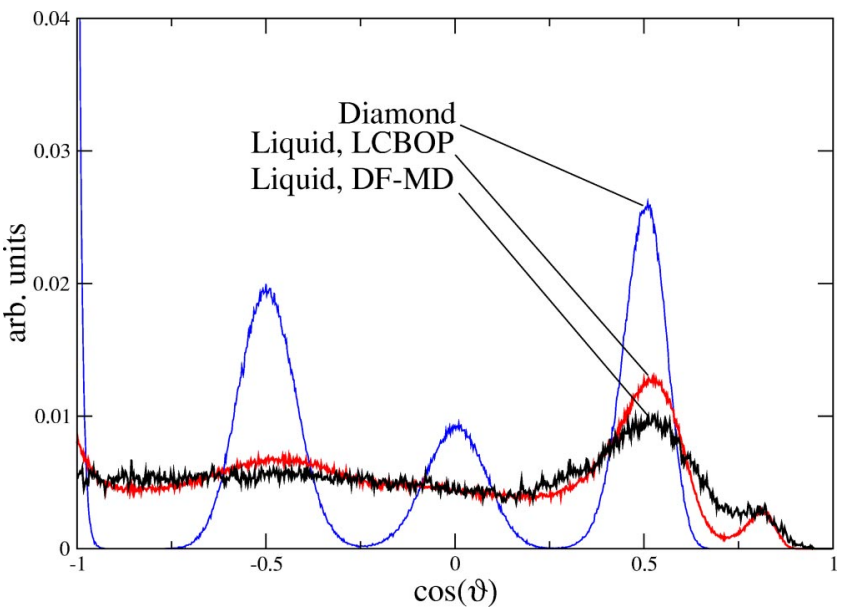

FIG. 5. (Color online) Angular correlation functions for second neighbors (see text) with state parameters as in Fig. 3. The peaks for the diamond are spread around the theoretical values for an fcc lattice of $-1,-0.5,0,0.5$, with weights $6 / 66,24 / 66,12 / 66,24 / 66$.

mond. The $g(r)$ 's obtained by LCBOP globally agree fairly well with those obtained by DF-MD, ${ }^{22}$ except around $2 \AA$, where the LCBOP minimum is too deep. In Fig. 4 we present the calculated angular correlation $g^{(3)}(\theta)$ for first neighbors, i.e., those atoms that fall within the short-range cutoff of the LCBOP. Again, the first shell of neighbors in the liquid has a strong tetrahedral ordering, comparable to bulk diamond. To test how far the local diamond structure persists in the liquid, we define the angular correlation function for second neighbors (i.e., all those particles that are first neighbors of the first neighbors, excluding the central atom and the first-neighbor shell). Figure 5 shows that, in the second-neighbor shell, the diamond structure is completely lost. Yet, the angular distribution is not structureless: we find a peak around $60^{\circ}$ and a shoulder at $\sim 35^{\circ}$; in a diamond lattice, the latter feature can be attributed to cross correlations between the first- and the second-neighbor shells.

It is rather surprising that the LCBOP reproduces the transformation to a predominantly fourfold coordinated liquid, while the REBO potential does not. Apparently, the isotropic long-ranged interactions play a crucial role. To demonstrate this, we verified that the short-ranged CBOP reproduces the behavior of the REBO potential. ${ }^{9}$ This behavior is rather puzzling as long-range interactions are expected to play a negligible role at these high densities (see, e.g., Glosli and Ree ${ }^{13}$ ). Moreover, long-ranged interactions were introduced in Los and Fasolino9 to describe threefold coordinated graphitic phases, and no attempt was made to make the long-range interactions dependent on the local environment. Torsional interactions appear to be important, since, without them, the calculated pressures would be too high for high densities and too low at low densities.

We conjecture that the combination of torsional interactions, and long-range forces is required to give the best description of the liquid. It would be interesting to check this conjecture by studying the liquid with other empirical potentials, in particular with Marks' environment-dependent interaction potential (EDIP). ${ }^{27}$ The study of the liquid with this 
potential presented in Marks $^{28}$ is limited to volumes of $6.2 \AA^{3}$ /atom where it gives coordination fractions comparable to those obtained here by DF and by LCBOP and in $\mathrm{Wu}$ et $a .^{8}$

In summary, on the basis of DF-MD simulations, we predict that liquid carbon should exhibit a continuous transformation from a threefold to a mostly fourfold coordinated liquid at high pressure. This liquid has a strong local angular ordering, reminiscent of the diamond structure. We have compared this finding with the results of MC simulations based on two empirical bond-order potentials with torsional terms, the short-range REBO potential and the long-range LCBOP. We find that the latter reproduces quite well the structure of both low-density and high-density liquid. The REBO simulations instead display a marked first-order phase transition between a mostly twofold and a mostly threefold graphite fluid in this range. It is tempting to speculate that the existence of a fourfold coordinate liquid at high densities will greatly facilitate the nucleation of diamonds from dense liquid carbon.

This work is part of the research program of the "Stichting voor Fundamenteel Onderzoek der Materie (FOM)," which is financially supported by the "Nederlandse Organisatie voor Wetenschappelijk Onderzoek (NWO)." E.J.M. acknowledges the Royal Netherlands Academy of Art and Sciences for financial support. J.L. and A.F. acknowledge NWO Project No. 015.000.031 for financial support. We acknowledge support from the Stichting Nationale Computerfaciliteiten (NCF) and the Nederlandse Organisatie voor Wetenschappelijk Onderzoek (NWO) for the use of supercomputer facilities.
${ }^{1}$ Y. Katayama, T. Mizutani, W. Utsumi, O. Shimomura, M. Yamakata, and K. Funakoshi, Nature (London) 403, 170 (2000).

${ }^{2}$ G. Franzese, G. Malescio, A. Skibinsky, S.V. Buldyrev, and H.E. Stanley, Nature (London) 409, 692 (2001).

${ }^{3}$ A. Ferraz and N.H. March, Phys. Chem. Liq. 8, 289 (1979).

${ }^{4}$ M. van Thiel and F.H. Ree, Phys. Rev. B 48, 3591 (1993).

${ }^{5}$ D.W. Brenner, Phys. Rev. B 42, 9458 (1990).

${ }^{6}$ D.W. Brenner, J.A. Harrison, C.T. White, and R.J. Colton, Thin Solid Films 206, 220 (1991).

${ }^{7}$ J.N. Glosli and F.H. Ree, Phys. Rev. Lett. 82, 4659 (1999).

${ }^{8}$ C.J. Wu, J.N. Glosli, G. Galli, and F.H. Ree, Phys. Rev. Lett. 89, 135701 (2002).

${ }^{9}$ J.H. Los and A. Fasolino, Phys. Rev. B 68, 024107 (2003).

${ }^{10}$ D.W. Brenner et al., J. Phys.: Condens. Matter 14, 783 (2002).

${ }^{11}$ F.P. Bundy, J. Chem. Phys. 38, 618 (1963).

${ }^{12}$ M. Togaya, Phys. Rev. Lett. 79, 2474 (1997).

${ }^{13}$ J.N. Glosli and F.H. Ree, J. Chem. Phys. 110, 441 (1999).

${ }^{14}$ M.P. Tosi, J. Phys.: Condens. Matter 6, A13 (1994).

${ }^{15}$ M.P. Grumbach and R.M. Martin, Phys. Rev. B 54, 15730 (1996).

${ }^{16}$ R. Car and M. Parrinello, Phys. Rev. Lett. 55, 2471 (1985).
${ }^{17}$ CPMD, J. Hutter, A. Alavi, T. Deutsch, M. Bernasconi, S. Goedecker, D. Marx, M. Tuckerman, and M. Parrinello, MPI für Festkörperforschung and IBM Zurich Research Laboratory, 1995-1999.

${ }^{18}$ W.G. Hoover, Phys. Rev. A 31, 1695 (1985).

${ }^{19}$ A.D. Becke, Phys. Rev. A 38, 3098 (1988).

${ }^{20}$ J.P. Perdew, Phys. Rev. B 33, 8822 (1986); Phys. Rev. B 34, 7406(E) (1986).

${ }^{21}$ P.E. Blöchl and M. Parrinello, Phys. Rev. B 45, 9413 (1992).

${ }^{22}$ J.H. Los et al. (unpublished).

${ }^{23}$ K. Raghavachari and J.S. Binkley, J. Chem. Phys. 87, 2191 (1987).

${ }^{24}$ K. Kobayashi, N. Kurita, H. Kumahora, and K. Tago, Phys. Rev. B 45, 11299 (1992).

${ }^{25}$ P.G. Dacosta, O.H. Nielsen, and K. Kunc, J. Phys. C 19, 3163 (1986).

${ }^{26}$ J.R. Morris, C.Z. Wang, and K.M. Ho, Phys. Rev. B 52, 4138 (1995).

${ }^{27}$ N.A. Marks, Phys. Rev. B 63, 035401 (2001). The EDIP potential takes into account interactions within a radius of $3.2 \AA$.

${ }^{28}$ N. Marks, J. Phys.: Condens. Matter 14, 2901 (2002). 